\title{
Chemical approaches to studying stem cell biology
}

\author{
Wenlin $\mathrm{Li}^{1}$, Kai Jiang ${ }^{2}$, Wanguo $\mathrm{Wei}^{3}$, Yan $\mathrm{Shi}^{4}$, Sheng Ding ${ }^{2}$ \\ ${ }^{I}$ Department of Cell Biology, Second Military Medical University, Shanghai 200433, China; ${ }^{2}$ Gladstone Institute of Cardiovascu- \\ lar Disease, University of California, San Francisco, CA 94158, USA; ${ }^{3}$ Stem Cell and Regenerative Medicine Center, Shanghai \\ Advanced Research Institute, Chinese Academy of Science, Shanghai 201210, China; ${ }^{4}$ Laboratory of Chemical Genomics, School \\ of Chemical Biology and Biotechnology, Shenzhen Graduate School of Peking University, Shenzhen, Guangdong, 518055, China
}

Stem cells, including both pluripotent stem cells and multipotent somatic stem cells, hold great potential for interrogating the mechanisms of tissue development, homeostasis and pathology, and for treating numerous devastating diseases. Establishment of in vitro platforms to faithfully maintain and precisely manipulate stem cell fates is essential to understand the basic mechanisms of stem cell biology, and to translate stem cells into regenerative medicine. Chemical approaches have recently provided a number of small molecules that can be used to control cell selfrenewal, lineage differentiation, reprogramming and regeneration. These chemical modulators have been proven to be versatile tools for probing stem cell biology and manipulating cell fates toward desired outcomes. Ultimately, this strategy is promising to be a new frontier for drug development aimed at endogenous stem cell modulation.

Keywords: small molecules; stem cells; self-renewal; differentiation

Cell Research (2013) 23:81-91. doi:10.1038/cr.2012.182; published online 25 December 2012

\section{Introduction}

Embryonic stem cells (ESCs) are derived from the inner cell mass of the preimplantation blastocyst. In vitro cultured ESCs are able to self-renew indefinitely and differentiate into the cells of the three primary germ layers (mesoderm, endoderm and ectoderm) of the body and even germ cells $[1,2]$. Thus, ESCs are an excellent platform for elucidating developmental biology, and are a valuable source for generating scarce or inaccessible cell types for therapeutic applications. Recent breakthroughs in stem cell biology, especially the induced pluripotent stem cell (iPSC) technology as well as induced lineage reprogramming by similar approaches [3-7], have opened up a new avenue to generate patient-customized functional cells for disease modeling and potential autologous transplantation without the challenges and controversies associated with using human ESCs (hESCs). In addition to the research activities toward pluripotent stem cells (PSCs), including both iPSCs and hESCs, significant efforts are also required to understand the bi-

Correspondence: Sheng Ding

Tel: 415-734-2717; Fax: 415-355-0141

E-mail: sheng.ding@gladstone.ucsf.edu ology of multipotent somatic stem cells, the endogenous cell populations dedicated to maintaining tissue homeostasis and mediating repair and regeneration. Dissection of the mechanisms that maintain pluripotency/multipotency and direct lineage specification is essential for the eventual applications of human PSCs and somatic stem cells. Chemical approaches, the discovery and use of functional small molecules to understand and manipulate biological systems, have proven to be useful tools for many biological discoveries [8]. Specifically in the stem cell field, chemical approaches are becoming powerful strategies to interrogate unknown pathways governing stem cell fate and to facilitate robust cell fate conversion. Compared with traditional genetic methods, which have been widely used for understanding the biological systems, chemical approaches offer distinct advantages. For example, small molecules typically provide a high degree of temporal control to rapidly inhibit or activate the function of specific proteins, the effects of which are often reversible, while traditional genetic methods are arduous and often involve permanent genetic modifications and complex late effects. In addition, the effects of small molecules can be finely tuned by delicately varying the concentrations or combinations of the small molecules. In this review, we will discuss the chemical approaches to the study of stem cell biology. It is not intended to be 
a comprehensive collection, but rather we will focus on studies that illustrate the strategies of chemical approaches to stem cell biology.

\section{Discovery-based chemical approaches}

Despite rapid advances in the field, stem cell research is still in its early stages. For example, chimeracompetent ESCs have only been derived from rodents. It is still very challenging to derive authentic ESCs from other highly valuable species, including livestock and non-human primates. Currently, our understanding of the different types of pluripotency during development is still very limited. The impact of species (and genetic background) difference on pluripotency is also largely unknown. In addition, our biological understanding of most somatic stem cells is still very limited, even for the extensively studied hematopoietic stem cells (HSCs). Because somatic stem and progenitor cells persist in many adult tissues and play essential roles in the maintenance of tissue homeostasis, dissection of the mechanisms regulating their fate (e.g., quiescence, activation, selfrenewal, migration, differentiation, etc.) will surely improve our understanding of tissue repair/regeneration and degeneration, and lead to development of better therapies for treating various diseases. Discovery-based chemical approaches, typically through phenotypic screenings of small molecule libraries, offer unique opportunities to identify useful tools to modulate stem cells and interrogate the underlying biology. Phenotypic chemical screening is a high-throughput chemical approach to discover compounds that enable the induction of a desired phenotype (e.g., a phenotypic change in cells or organisms). It is an unbiased approach because it probes overall mechanisms (including signaling pathways and epigenetic mechanisms, etc.) without requiring much of prior knowledge of the target(s) being modulated (Figure 1). This approach is especially suitable to initiate discovery in stem cell biology. In this section, we will discuss the strategies and discoveries made using discovery-based chemical approaches, which have been critical to solving otherwise intractable issues and revealing previously un-

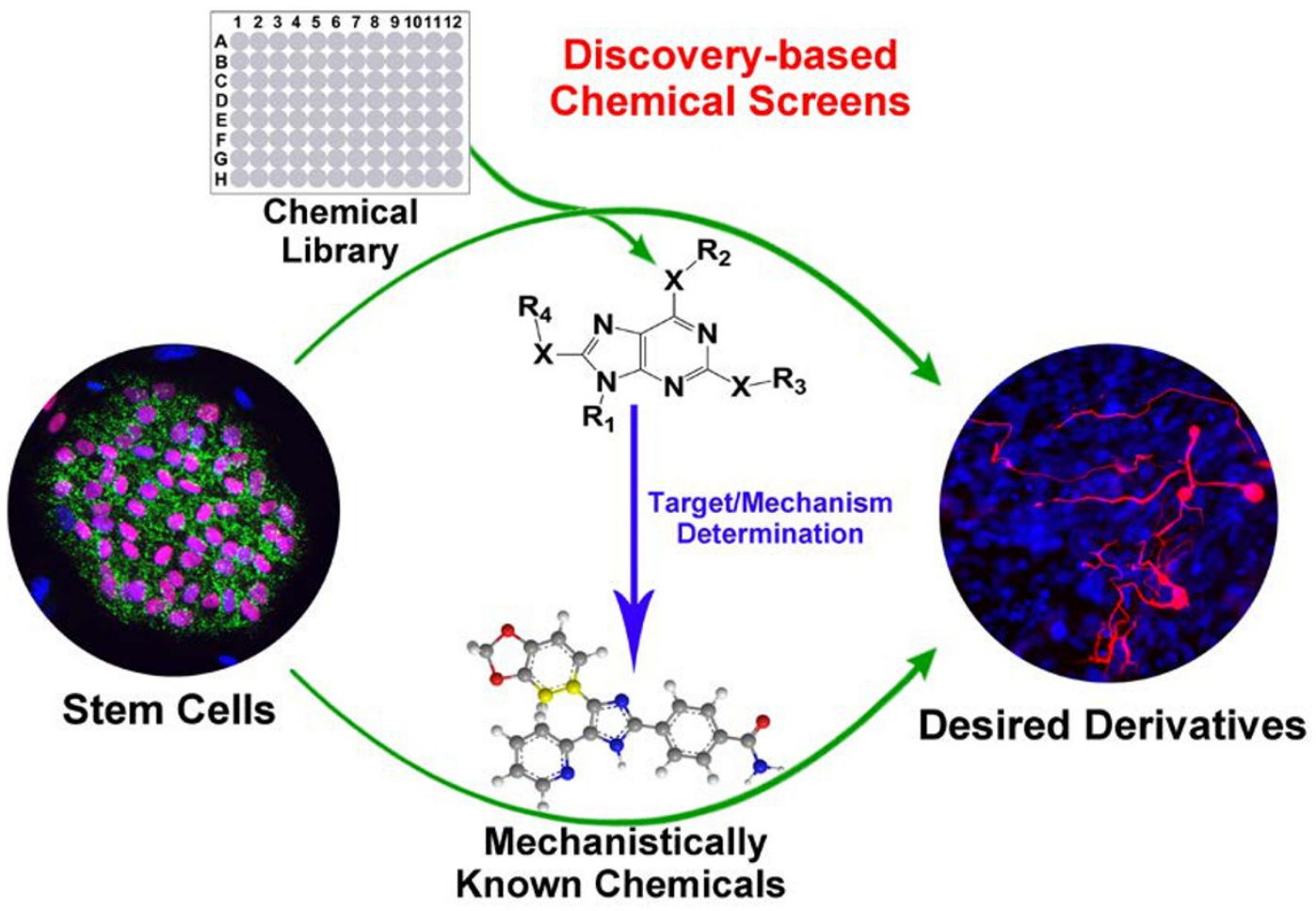

\section{Rationale-based Chemical Approach}

Figure 1 Scheme of chemical approaches to modulating cell fates. Discovery-based phenotypic screening is a powerful strategy to identify new chemical modulators of stem cell fate and to elucidate the underlying mechanisms. In contrast, the rationale-based chemical approach is hypothesis-driven, and requires the prior knowledge of stem cell and developmental biology to devise strategies to modulate stem cell fate by using mechanistically known small molecules. 
known mechanisms in stem cell biology.

Leukemia inhibitory factor (LIF)-signal transducer and activator of transcription 3 (STAT3) and bone morphogenetic protein 4 (BMP4)-SMAD signaling are well characterized pathways essential for maintaining mESC self-renewal $[9,10]$. However, whether or not there are other deterministic mechanisms was unknown. From a high-throughput screening of novel small molecule libraries, we identified a new 3,4-dihydropyrimido (4,5d) pyrimidine analog named pluripotin/SC1 that could maintain mESCs in the undifferentiated, pluripotent state under chemically defined conditions in the absence of feeder cells, serum, LIF and BMP4 [11]. Mechanism studies revealed that pluripotin directly interacts with and inhibits the differentiation-inducing signaling components extracellular signal-regulated kinases (ERK) and Ras GTPase activating protein, without affecting the classic mESC self-renewal pathways, including LIF/ STAT3, BMP4/SMAD or $\mathrm{Wnt} / \beta$-catenin signaling. This study provided a conceptual advance that $\mathrm{mESC}$ self-renewal can be established intrinsically through inhibiting the differentiation-inducing signaling, and highlighted the power of chemical approaches in dissecting the complex biology of stem cells. Currently, a number of compounds that can enhance stem cell self-renewal, survival, differentiation and reprogramming have been identified through this discovery-based chemical approach and these findings have significantly advanced stem cell research and the applications thereof.

Aside from the self-renewal of PSCs, expansion of most types of somatic stem cells, which are directly applicable to regenerative medicine, remains a technical challenge. Considering that many somatic stem cells selfrenew persistently in vivo, the failures to expand them in vitro reflect our currently limited understanding of their complex in vivo microenvironment, also called the stem cell niche [12]. Before the thorough molecular dissection of the underlying mechanisms, which is essential to rationally devise appropriate conditions for somatic stem cell modulation, phenotype screening (e.g., using the phenotype of stem cell expansion as a readout) of small molecule libraries represents a feasible way to identify and then mechanistically characterize small molecules that promote self-renewal of somatic stem cells. For example, by using the expression of CD34 and CD133 as a readout to screen a library of 100000 heterocycles, Boitano et al. [13] identified a purine derivative, StemRegenin 1 (SR1), that can promote the ex vivo expansion of primary CD34 positive human HSCs. In contrast to standard HSC expansion using serum-free media with cytokines, including SR1 in the media with cytokines led to an additional 50-fold expansion of CD34 positive cells, and a 17-fold increase in cells that can functionally repopulate the hematopoietic system of NOD/SCID mice. Notably, SR1 alone cannot induce HSC expansion, and SR1 plus cytokines does not alter cell division rate compared with the cytokine only treatment, suggesting that SR1 may augment CD34 positive HSC populations mainly by preventing their differentiation. Mechanistic studies show that SR 1 functions through directly antagonizing the aryl hydrocarbon receptor, which had never been reported to modulate HSC self-renewal before. This study again reinforces the notion that unbiased phenotypic screening is useful to probe the unknown mechanisms in stem cell biology. Practically, an improved capacity to expand these therapeutically valuable stem cells, such as HSCs, will surely alleviate the serious problems associated with the shortage of donor cells. A more recent study from the same group identified a naphthyridinone (MK1) that induces the differentiation of primary human CD34 positive cells to megakaryocytes by screening a library of 50 000 heterocycles using CD41, a selective megakaryocyte marker, as a readout. FACS analysis suggested that MK1 facilitated the induction of megakaryocyte/erythrocyte progenitors from common myeloid progenitors at the expense of granulocyte/monocyte progenitors. Functional studies revealed that the compound acts through inhibition of platelet-derived growth factor receptor signaling in common myeloid progenitors. Molecules like this may have a utility in recovering platelets after high-dose chemotherapy [14].

For hESC research and application, one of the significant obstacles is the poor cell survival after single cell dissociation. Recently, Sasai's group screened a panel of growth factors and small molecule modulators of apoptosis-related enzymes and kinases in dissociated hESCs. Small molecule Y-27632, a selective Rho-associated kinase (ROCK) inhibitor was identified [15], which is able to promote survival of single-cell-dissociated hESCs without the loss of pluripotency. To gain a better understanding of the molecular mechanisms governing hESC survival, we performed a high-throughput phenotypic screening of 50000 novel synthetic compounds to identify small molecules that allow hESC survival after trypsin dissociation [16]. Two distinct potent compoundsa 2,4-disubstituted thiazole (named thiazovivin/Tzv) and a 2,4-disubstituted pyrimidine (named Pyrintegin/Ptn) were identified to significantly increase single-cell survival with enhanced self-renewal. Interestingly, Tzv can promote cell survival in both adherent and suspension culture conditions, while Ptn is only effective in adherent culture conditions. Affinity pull-down experiments using a Tzv-immobilized matrix (along with other studies) revealed that Tzv enhances E-cadherin stability and cell- 
cell interactions through the inhibition of ROCK, while Ptn regulates cell-ECM (extracellular matrix) interactions to indirectly enhance E-cadherin stability. At the cell surface, the E-cadherin-mediated cell-cell interactions and integrin-mediated cell-ECM interactions form a positive feedback regulatory loop via inhibition of Rho-ROCK signaling pathways to regulate the survival of hESCs. Upon cell dissociation, Rho-ROCK signaling is up-regulated, resulting in the destabilization of Ecadherin, cytoskeletal hypercontraction, and decreased cell adhesion ability. Loss of cell-cell adhesion, in turn, further increases Rho-ROCK activity. This positive autoregulation ultimately results in the hyperactivation of ROCK signaling and irreversible disruption of cell-cell and cell-ECM adhesion. Two concurrent studies revealed that hESC dissociation (breakdown of E-cadherinmediated interaction) led to long-lasting apoptotic cellular contraction due to myosin hyperactivation in a Rho/ ROCK-dependent manner. Inhibition of ROCK or myosin heavy chain ATPase (by blebbistatin) diminishes actomyosin contraction and rescues the dissociated hESCs from apoptosis $[17,18]$.

Differentiation of hESCs into desired functional cell types is another fundamental issue in order to unleash their therapeutic potential. While enormous progresses have been made over the years on ESC differentiation into a wide variety of cell types [19-23], significant improvements are still required to generate target cells more rapidly, efficiently and safely under defined conditions for potential clinic applications. In vitro ESC differentiation is largely a process that recapitulates normal in vivo lineage development [24-28]. However, due to the intrinsic complexities and our limited understanding of these processes, it is not always straightforward to devise the step-wise differentiation in vitro. Phenotypic screening offers a way to tackle such intractable puzzles in hESC differentiation. Low efficiency is a bottleneck for many step-wise lineage differentiation methods, which typically depend on various developmental morphogens. The concentration of morphogens is usually critical for the outcome of differentiation. However, the short life of protein factors in the media and their variable potency could compromise the efficiency and reproducibility of lineage differentiation. Moreover, protein regimens are very costly, especially in terms of large-scale cell culture. Discoveries of small molecules that can replace or enhance the effects of morphogen factors would facilitate the development of more reproducible differentiation paradigms. Using the expression of Sox 17 as a readout, Zhu et al. [29] recently screened 20000 small molecules for their ability to enhance mESC differentiation toward endoderm under low concentrations of activin A, a morphogen widely used to induce endoderm at high concen- trations. A compound, named stauprimide, was identified to promote efficient induction of Sox 17 in both mouse and human ESCs synergistically with low concentrations of activin A, although stauprimide alone does not have the effect. Target identification revealed that stauprimide can interact with NME2, a c-Myc-activating transcription factor, and inhibit its nuclear localization. Upon stauprimide treatment, the resulted down-regulation of c-Myc may destabilize the self-renewal state of ESCs, which renders ESCs more susceptible to differentiation cues. Indeed, further studies showed that stauprimide enhances not only endoderm differentiation of ES cells, but also differentiation toward mesoderm and ectoderm lineages under appropriate lineage-specifying conditions. Another study used Sox 17 promoter-driven dTomato as a reporter, and screened a collection of 4000 compounds for small molecules that could induce definitive endoderm in the absence of activin A. Two structurally similar small molecules, IDE1 and IDE2, were found to induce endoderm differentiation in up to $80 \%$ of mESCs (or $50 \%$ of hESCs) in the absence of activin A [30]. Similar to activin A, both IDE1 and IDE2 induce SMAD2 phosphorylation in mESCs although their molecular targets remain unknown. Another more recent study used reporter monkey ESCs that express EGFP driven by the human alpha myosin heavy chain promoter to screen small molecules that promote cardiac differentiation using a defined, cytokine-free media supplemented with glycogen synthase kinase 3 (GSK3) inhibitors. They found a small molecule (KY02111) that when applied on days 4-8 of induction could increase GFP expression, whereas treatment on days 0-4 completely repressed GFP expression. Preliminary data suggested that KY02111 suppressed Wnt signaling downstream GSK3 $\beta$ in the canonical Wnt signaling pathway, but its precise target remains unknown [31]. Similarly, another study showed that treatment for $48 \mathrm{~h}$ by high concentrations of the GSK3 inhibitor CHIR99021 can induce highly efficient primitive streak induction from hESCs. Using the generated brachyury positive primitive streak population as the starting cells for a cardiac induction screening, IWR-1endo, a tankyrase inhibitor that can inhibit Wnt signaling through stabilizing axin, was identified from a collection of 300 known signal transduction modulators to enhance cardiac differentiation [32]. These studies suggest that modulation of Wnt signaling in a precise time window is essential for efficient cardiac differentiation. Further efforts to identify chemical modulators of essential developmental pathways will surely facilitate the development of more robust and efficient PSC differentiation methods.

Recent advances in using over-expression of defined transcription factors (e.g., Oct4, Sox2, Klf4, and c-Myc) 
to reverse lineage-committed cells into a pluripotent state represent a major breakthrough in cellular reprogramming [3-7]. Although somatic cell nuclear transfer and cell fusion have been used to reprogram lineagerestricted cells to the totipotent (or pluripotent) state, somatic cell nuclear transfer was technically complicated and ethically controversial for human study; and cell fusion-generated heterokaryons are polyploid harboring an additional set of chromosomes. The iPSC technology is remarkably simple, which has enabled its widespread utilizations in the generation of individual-specific PSCs. However, ectopic transcription factor over-expression could result in integration of exogenous DNA fragments into the host cell genome. Although significant progresses have been made to generate iPSCs without genomic manipulation by a variety of approaches [3338], these approaches usually substantially compromise reprogramming efficiency and kinetics. As the cells are undergoing drastic phenotype changes during reprogramming, phenotypic chemical screenings can be easily adapted to identify chemical compounds that function in reprogramming. In the past several years, a number of small molecules and their combinations were identified that can functionally substitute for reprogramming transcription factors, enhance reprogramming efficiency, accelerate reprogramming speed, and that are useful for revealing mechanisms underlying the reprogramming process.

Using phenotypic screening with Oct4 promoter-driven GFP expression and colony morphology as the selection criteria, we identified BIX-01294, a small-molecule inhibitor of the H3K9 histone methyltransferase G9a [39], that enabled reprogramming with ectopic expression of only Oct4 and Klf4 in somatic cells. When BIX-01294 was combined with a DNA methyltransferase inhibitor RG108 or an L-type calcium channel agonist Bayk-8644, fibroblasts could be as efficiently reprogrammed using only Oct4 and Klf4 as with the original four transcription factors [40, 41]. Remarkably, BIX-01294 could substitute for Oct 4 when treating neural progenitor cells transduced with Sox2, Klf4 and c-Myc. This effect is consistent with previous findings revealing that repressive $\mathrm{H} 3 \mathrm{~K} 9$ methylation is associated with the silencing of pluripotency genes (such as Oct3/4 and Rexl) during differentiation [42]. BIX-01294 may therefore function to facilitate an epigenetic shift toward pluripotency by unsilencing Oct4 to promote its active transcription. Other chemical screenings identified histone deacetylase inhibitors, including valproic acid (VPA) and sodium butyrate, as compounds able to promote mouse and human somatic cell reprogramming [43-45]. In particular, VPA enabled human fibroblasts to be reprogrammed with two factors
(Oct4 and Sox2) [43], and facilitated the reprogramming of mouse fibroblasts into iPSCs using recombinant proteins of the original reprogramming transcription factors [37]. In addition, parnate (an inhibitor of the H3K4/9 histone demethylase LSD1) and EPZ004777 (an inhibitor of the histone H3K79 methyltransferase DOT1-like) were identified as agents promoting the reprogramming of human cells $[46,47]$. All these studies suggest that remodeling of the somatic epigenetic landscape represents a major reprogramming barrier.

Through a chemical screening, PS48, a small-molecule allosteric activator of 3' phosphoinositide-dependent kinase 1, was identified to enable the reprogramming of human adult keratinocytes, umbilical vein endothelial cells, or amniotic fluid-derived cells by Oct4 alone [48]. Mechanism studies revealed that PS48 exerts its effects by enhancing glycolysis at the early stage of reprogramming. hESCs are highly dependent on glycolytic metabolism $[49,50]$, which is advantageous for highly proliferative cells and stem cells by more effectively producing various macromolecular precursors to meet the demands of rapid biosynthesis while generating fewer reactive oxygen species that can induce oxidative damage. This study suggests that metabolic conversion from mitochondrial oxidation to glycolysis represents another fundamental mechanism operant during iPSC reprogramming.

As an unbiased strategy, discovery-based chemical approaches are especially versatile. In addition to applying this strategy to manipulate cell fate as discussed, phenotypic chemical screenings have also been successfully applied in dissecting entangled mechanisms under more specific physiological or pathological settings. To mimic the neurotoxicity of microglial activation, a recent study established an assay system by adding interferon- $\gamma$ - and lipopolysaccharide-activated BV2 cells (a microgalial cell line) into the co-culture of stem cell-derived motor neurons and astrocytes. 10000 small molecules were screened using this assay system, and a number of neuroprotective small molecules were identified that prevented the neurons in the co-culture system from degeneration through diverse mechanisms, including inhibition of nitric oxide production by microglia, stimulation of the nuclear factor erythroid 2-related factor 2 pathway in microglia and astrocytes, and direct protection of neurons from nitric-oxide-induced cell death [51]. Approaches like this represent a promising strategy to discover lead compounds for drug development.

\section{Rationale-based chemical approaches}

The knowledge gained from developmental biology provides clues and hypotheses in modulating stem cell 
fates in vitro by recapitulating normal developmental processes. Well characterized small molecules, especially those that can target essential embryonic signaling pathways, are becoming indispensible tools to manipulate stem cells either alone or in combination with other protein factors (Figure 1). Distinct from the aforementioned discovery-based chemical approaches, these rationalebased chemical approaches are hypothesis-driven and require prior knowledge of stem cell and developmental biology. In this section, we will illustrate some representative works that used function-known small molecules to modulate cell fate and probe stem cell biology.

Previous studies suggested that the ERK/mitogenactivated protein kinase (MAPK) pathway plays a negative role in mESC self-renewal [11, 52]. Based on this prior knowledge, Ying et al. [53] found that application of MAPK/ERK kinase (MEK) and/or fibroblast growth factor (FGF) receptor inhibitors can prevent mESC differentiation under defined conditions without LIF supplementation. However, this condition cannot sustain robust mESC proliferation and survival. Addition of another small molecule inhibitor of GSK3 (CHIR99021 in this case), which had previously been shown to support ESC self-renewal, results in the maintenance of a "ground state" self-renewal without activating classic self-renewal pathways including LIF/STAT3 and BMP4/SMAD pathways. This study supported the notion that mESCs have an "innate" ability to self-renew when shielded from differentiation-inducing signals [11]. Similarly, the combinations of factors that inhibit differentiation signaling pathways in conjunction with LIF supported the derivation and long-term self-renewal of mESCs from refractory strains $[54,55]$, rat ESCs and rat induced pluripotent stem cells [56-59].

Comared to mESCs, hESCs display significant differences in terms of colony morphology, gene expression pattern, and the signaling responses in self-renewal and differentiation. hESCs are usually cultured with basic FGF (bFGF) and require the activation of MAPK and transforming growth factor $\beta$ (TGF $\beta$ )/activin/nodal pathways for self-renewal. hESCs do not respond to LIF/ STAT3 signaling, and BMP4 induces their differentiation. Interestingly, hESCs highly resemble the so called epiblast stem cells (EpiSCs). In contrast to mESCs from and representing the inner cell mass of preimplantation mouse blastocyst, EpiSCs were derived from the postimplantation egg cylinder-stage epiblasts $[60,61]$. These observations suggest that the pluripotency of hESCs may be developmentally distinct from mESCs, and raise the interesting question of whether a mESC-like, early stage of pluripotency can be established for human pluripotent stem cells. By combining genetic reprogramming and cell signaling modulation with small molecules that favor the mESC-like state, mESC-like, naïve-state human iPSCs (m-hiPSCs) can be generated from human fibroblasts by ectopic expression of Oct4, Sox2, Nanog, and Lin28, in culture medium that contains human LIF and the combination of three chemicals, PD0325901, A-83-01, and CHIR99021 [56]. A-83-01 is a small molecule inhibitor of the TGF $\beta$ /activin receptors. m-hiPSCs form domed colonies and exhibit stable long-term selfrenewal under the condition with hLIF and the small molecule combination, suggesting that m-hiPSCs are distinct from hESCs in signaling responses and self-renew in the mESC-like state. Similarly, another study showed that ectopic expression of Oct4, Klf4, and Klf2 combined with LIF and CHIR99021/PD0325901 can convert hESCs into a mESC-like state [62]. However, whether mESC-like hESCs can be derived from human blastocysts without any ectopic gene expression still needs to be further investigated.

PSC differentiation is another field where rationalebased chemical approaches are especially effective. Pioneering works of ESC differentiation demonstrated that knowledge gained from in vivo developmental biology provides a basis in devising lineage specification strategies $[24,63]$. Given their advantages over protein factors as discussed, small molecules modulating critical developmental signaling pathways have been studied and used to control stem cell differentiation. For example, TGF $\beta$ signaling is important for hESC self-renewal, and mesoderm/endoderm induction. Inhibition of TGF $\beta$ signaling, for example by SB431542 (a small molecule inhibitor of activin receptor-like kinase 4, 5 and 7), favors/facilitates neural induction of hESCs [64]. Recently, Chambers et al. [65] developed an efficient neural induction method for hESCs in a monolayer fashion by the combination of Noggin (a secreted protein that binds to and inhibits BMP4) and SB431542. Following-up studies showed that a small molecule BMP receptor inhibitor can fully replace Noggin for efficient neural induction together with SB431542 [66]. These two pathway inhibitors might function synergistically to destabilize self-renewal, to induce neural lineage and to prevent cells from differentiating into trophectoderm, mesoderm and endoderm lineages (on which BMP and TGF $\beta$ signaling have inductive effects).

In addition to generating differentiated cells, capturing/maintaining the intermediate stem/progenitor cell population during ESC differentiation is highly significant. We recently identified combinations of small molecules for the induction or expansion of pre-rosette stage neural stem cells (primitive neural stem cells, pNSCs) from hESCs in culture [67]. We found that combining 
a GSK3 inhibitor (i.e., CHIR99021) with TGF $\beta$ and Notch signaling pathway inhibitors induced a highly efficient conversion of monolayer-cultured hESCs into homogenous primitive neuroepithelia within one week. Remarkably, the combination of LIF, CHIR99021 and SB431542 effectively captured and maintained these pNSCs for long-term self-renewal in vitro. The underlying mechanisms of this strategy mainly involve the synergy of 1) TGF $\beta$ /activin pathway inhibition that induces neuralization of hESCs by destabilizing pluripotency and blocking mesendoderm differentiation, and 2) GSK3 inhibition and LIF that promote pNSC selfrenewal $[68,69]$. pNSCs are distinct from the typical bFGF-dependent neural precursor cells that exhibit glial differentiation propensity and loss of responsiveness to morphogen patterning. Notably, the long-term expanded pNSCs maintain their high neurogenic differentiation propensity and remain highly plastic and responsive to instructive regional patterning cues for differentiation toward midbrain and hindbrain neuronal subtypes. As it is practically impossible to non-invasively isolate most types of adult stem cells from specific tissues, derivation and expansion of adult stem cells from PSCs represent an attractive alternative approach. Applying such a strategy to other lineages and developmental stages is a novel area of research that could lead to the development of new cell-based therapies.

Although the detailed mechanisms of reprogramming to pluripotency are largely unknown, reprogramming is concomitant with dramatic cellular changes (e.g., changes in gene expression, morphology, and metabolism), which may be required for, or even represent barriers to, successful reprogramming. Rather than being simply consequences of the reprogramming process, regulating these changes directly may modulate the reprogramming process. For example, reprogramming fibroblasts into iPSCs entails a mesenchymal-to-epithelial transition (MET). Therefore, it would be tempting to test if small molecules that facilitate the MET process could enhance reprogramming. Indeed, small molecules that target three known MET-related mechanisms, including inhibition of TGF $\beta$ receptors (by SB431542), MEK (by PD0325901), or ROCK (by thiazovivin) significantly enhanced reprogramming efficiency and accelerated reprogramming speed when added individually or in combination, at least in part by derepressing the epithelial phenotype via up-regulation and stabilization of E-cadherin expression [70]. In addition, the initiation of reprogramming has been shown to elicit a stress response resulting in cellular senescence, as demonstrated by observations of upregulation of p53 and p21 expression and accumulation of reactive oxygen species shortly after the transduction of reprogramming transcription factors. Thus compounds that alleviate cellular senescence may promote reprogramming. Consistently, it was found that vitamin $\mathrm{C}$ can enhance reprogramming in part by reducing p53 expression and alleviating senescence [71]. A recent study further showed that vitamin $\mathrm{C}$ promoted reprogramming also by modulating Jhdm1a/1b, two known vitamin Cdependent $\mathrm{H} 3 \mathrm{~K} 36$ histone demethylases that are potent regulators of reprogramming [72]. Consistently, Jhdm1b activation accelerates cell cycle progression and suppresses cellular senescence by repressing the INK4/ARF locus. Another study demonstrated that aberrant epigenetic silencing of the imprinted Dlk1-Dio3 gene cluster, which was observed in some generated iPSC lines, could be prevented with vitamin $C$ treatment through indirectly interfering with Dnmt3a-mediated hypermethylation of the Dlk1-Dio3 locus [73]. The presence of vitamin C during reprogramming allowed the generation of high quality iPSCs even from mature B cells with the capacity to generate all iPSC-mice through tetraploid complementation [73].

\section{Chemical approaches modulating in vivo regenera- tion}

With the availability of appropriate cell sources, replacement of damaged cells is a straightforward solution for many degenerative diseases and tissue injuries. However, considering the tremendous challenges to develop, manufacture and transplant appropriate cells, an alternative approach is to pharmacologically modulate endogenous stem/progenitor cells for repair and regeneration. Recent progresses have adopted the chemical strategies outlined above to modulate the fates of endogenous stem cells.

Zebrafish, which can be cultivated in multi-well plates during various developmental stages, is a widely used model animal for chemical phenotypic screenings for various purposes, including induction of in vivo regeneration. North et al. screened a collection of biologically active compounds using zebrafish to identify modulators of HSC induction in the zebrafish aorta-gonadmesonephros region, from where the definitive HSCs are primarily initiated. Their work led to the discovery that small molecules that enhance prostaglandin E2 (PGE2) synthesis, including PGE2 itself, increased HSC numbers $[74,75]$. These results have been further confirmed by in vitro and in vivo studies in the murine model. This discovery has led to a rapid phase I clinical trial of ex vivo 16, 16-dimethyl-PGE2 (a more stable derivative of PGE2) treated umbilical cord blood units in a transplantation setting. Similar approaches using zebrafish have 
also been successful in identifying small molecules that can induce expansion of endogenous cardiac progenitor cells [76], and pancreatic beta cells [77]. In addition to the zebrafish model, Pieper et al. [78] recently reported a study, where an in vivo chemical screening of 1000 compounds was carried out to identify proneurogenic compounds that stimulate neurogenesis in the subgranular zone in mice. One of the hits, designated P7C3, was shown to promote survival of newborn neurons without apparently stimulating neural precursor cell proliferation. In vivo administration of $\mathrm{P} 7 \mathrm{C} 3$ rescued the morphological and electrophysiological deficits in dentate gyrus of the mice lacking Npas3, a critical transcriptional factor for neurogenesis. Prolonged treatment of aged rats with P7C3 enhanced neurogenesis and ameliorated cognitive decline. Although the precise target of $\mathrm{P} 7 \mathrm{C} 3$ remains unknown, P7C3 may function through inhibition of apoptotic cell death by preserving the mitochondrial membrane integrity [78]. Subsequent structure optimization generated more potent analogs of $\mathrm{P} 7 \mathrm{C} 3$ with improved pharmacokinetic properties [79]. These discovery-based in vivo chemical approaches represent powerful strategies to uncover discovery of new therapeutic targets and drug candidates.

Similarly, rationale-based chemical approaches have also been used to modulate cell fate in vivo. Neurofibromatosis type I (NF-1) is a human genetic disorder with an incidence of about 1 in 3500 live births. The most common complication of NF-1 patients is cognitive and learning disability associated with brain structural defects, such as enlarged corpus callosum. NF-1 is caused by loss-of-function mutations of the neurofibromin 1 gene, which encodes a RAS GTPase-activating protein that negatively regulates RAS/ERK signaling. Neurofibromin 1-deficient mice phenotypically recapitulate human NF-1 disease. Studies suggested that neurofibromin 1 mutations result in hyper-activation of RAS/ERK signaling, which promotes ectopic expression of Olig2, specifically in the subventricular zone type $\mathrm{C}$ neural progenitors, and shifts their differentiation propensity toward glial cells at the expense of neurogenesis in neonatal mice. Based on this knowledge, transient treatment of neurofibromin 1-deficient mice on postnatal days 0.5-18 with MEK inhibitor PD0325901 completely rescued the phenotypes of these mice by restoring the normal differentiation propensity of subventricular zone type $\mathrm{C}$ neural progenitors. This study indicates that MEK inhibition is a promising strategy to prevent and treat NF1 [80].

Stromal cell-derived factor 1 (SDF-1), which interacts with and signals through $\mathrm{C}-\mathrm{X}-\mathrm{C}$ chemokine receptor type 4 (CXCR4), chemoattracts hematopoietic stem and progenitor cells. The SDF-1/CXCR4 axis has been implicat- ed in the homing and retention of HSCs. CD26/dipeptidylpeptidase IV is a membrane-bound extracellular peptidase that cleaves and inactivates SDF-1. Previous studies showed that inhibition/deletion of CD26 enhanced the homing and engraftment of mouse long-term competitive repopulating $\mathrm{HSCs}$ in lethally irradiated congenic mice [81]. It is worth noting that the SDF-1/CXCR4 axis represents a general chemotactic mechanism not restricted to the bone marrow. For example, in ischemic heart tissue, SDF- $1 \alpha$ is the major chemokine attracting endogenous endothelial progenitors that express the SDF- $1 \alpha$ receptor CXCR4 and home to the injured heart [82]. Accordingly, Zaruba et al. recently described a small molecule-based regenerative strategy for treating myocardial infarction by recruiting endogenous bone marrow endothelial progenitors to the heart, which ultimately led to the generation of new blood vessels and improvement of heart functions [83]. The authors demonstrated that combined administration of granulocyte colony-stimulating factor (G-CSF) and the CD26 inhibitor Diprotin A can enhance the recruitment of CXCR4 positive stem cells to myocardium and improve survival and myocardial function by increasing neovascularization. Here, G-CSF was used to mobilize endothelial progenitors from the bone marrow. Although this study did not involve the direct targeting of endogenous stem cells by the small molecule, it represents an excellent example where a small molecule is used to facilitate the trafficking of stem cells to injury site for tissue repair. Similar strategy could be useful for other degenerative diseases using elaborately balancing the modulation of endogenous stem cell migration, expansion and differentiation using small molecules and/or protein factors to achieve tissue repair and regeneration.

\section{Summary and perspective}

Chemical approaches are powerful tools to manipulate stem cell fate, which facilitate not only improved generation of desired cell types, but also better understanding of the underlying molecular mechanisms. With new advances in stem cell research and chemical biology, this approach will be further strengthened by the decades of experiences in development of conventional pharmaceutical molecules. Prospectively, these molecules, identified in their crossover with stem cells, represent promising candidates in efforts to develop new drugs aimed to induce in vivo regeneration through modulating endogenous cells.

\section{References}

1 Smith AG. Embryo-derived stem cells: of mice and men. 
Annu Rev Cell Dev Biol 2001; 17:435-462.

2 Keller G. Embryonic stem cell differentiation: emergence of a new era in biology and medicine. Genes Dev 2005; 19:1129-1155.

3 Takahashi K, Tanabe K, Ohnuki M, et al. Induction of pluripotent stem cells from adult human fibroblasts by defined factors. Cell 2007; 131:861-872.

4 Takahashi K, Yamanaka S. Induction of pluripotent stem cells from mouse embryonic and adult fibroblast cultures by defined factors. Cell 2006; 126:663-676.

5 Okita K, Ichisaka T, Yamanaka S. Generation of germlinecompetent induced pluripotent stem cells. Nature 2007; 448:313-317.

6 Yu J, Vodyanik MA, Smuga-Otto K, et al. Induced pluripotent stem cell lines derived from human somatic cells. Science 2007; 318:1917-1920.

7 Wernig M, Meissner A, Foreman R, et al. In vitro reprogramming of fibroblasts into a pluripotent ES-cell-like state. $\mathrm{Na}$ ture 2007; 448:318-324.

$8 \mathrm{Xu}$ Y, Shi Y, Ding S. A chemical approach to stem-cell biology and regenerative medicine. Nature 2008; 453:338-344.

9 Niwa H, Burdon T, Chambers I, Smith A. Self-renewal of pluripotent embryonic stem cells is mediated via activation of STAT3. Genes Dev 1998; 12:2048-2060.

10 Ying QL, Nichols J, Chambers I, Smith A. BMP induction of Id proteins suppresses differentiation and sustains embryonic stem cell self-renewal in collaboration with STAT3. Cell 2003; 115:281-292.

11 Chen S, Do JT, Zhang Q, et al. Self-renewal of embryonic stem cells by a small molecule. Proc Natl Acad Sci USA 2006; 103:17266-17271.

12 Watt FM, Hogan BL. Out of Eden: stem cells and their niches. Science 2000; 287:1427-1430.

13 Boitano AE, Wang J, Romeo R, et al. Aryl hydrocarbon receptor antagonists promote the expansion of human hematopoietic stem cells. Science 2010; 329:1345-1348.

14 Boitano AE, de Lichtervelde L, Snead JL, Cooke MP, Schultz PG. An image-based screen identifies a small molecule regulator of megakaryopoiesis. Proc Natl Acad Sci USA 2012; 109:14019-14023.

15 Watanabe K, Ueno M, Kamiya D, et al. A ROCK inhibitor permits survival of dissociated human embryonic stem cells. Nat Biotechnol 2007; 25:681-686.

$16 \mathrm{Xu} \mathrm{Y,} \mathrm{Zhu} \mathrm{X,} \mathrm{Hahm} \mathrm{HS,} \mathrm{et} \mathrm{al.} \mathrm{Revealing} \mathrm{a} \mathrm{core} \mathrm{signaling}$ regulatory mechanism for pluripotent stem cell survival and self-renewal by small molecules. Proc Natl Acad Sci USA 2010; 107:8129-8134.

17 Ohgushi M, Matsumura M, Eiraku M, et al. Molecular pathway and cell state responsible for dissociation-induced apoptosis in human pluripotent stem cells. Cell Stem Cell 2010; 7:225-239.

18 Chen G, Hou Z, Gulbranson DR, Thomson JA. Actin-myosin contractility is responsible for the reduced viability of dissociated human embryonic stem cells. Cell Stem Cell 2010; 7:240-248.

19 Giudice A, Trounson A. Genetic modification of human embryonic stem cells for derivation of target cells. Cell Stem Cell 2008; 2:422-433.

20 Tian X, Kaufman DS. Differentiation of embryonic stem cells towards hematopoietic cells: progress and pitfalls. Curr Opin Hematol 2008; 15:312-318.

21 Spence JR, Wells JM. Translational embryology: using embryonic principles to generate pancreatic endocrine cells from embryonic stem cells. Dev Dyn 2007; 236:3218-3227.

22 Erceg S, Ronaghi M, Stojkovic M. Human embryonic stem cell differentiation toward regional specific neural precursors. Stem Cells 2009; 27:78-87.

23 Murry CE, Keller G. Differentiation of embryonic stem cells to clinically relevant populations: lessons from embryonic development. Cell 2008; 132:661-680.

24 Wichterle H, Lieberam I, Porter JA, Jessell TM. Directed differentiation of embryonic stem cells into motor neurons. Cell 2002; 110:385-397.

25 Li XJ, Du ZW, Zarnowska ED, et al. Specification of motoneurons from human embryonic stem cells. Nat Biotechnol 2005; 23:215-221.

26 Kroon E, Martinson LA, Kadoya K, et al. Pancreatic endoderm derived from human embryonic stem cells generates glucose-responsive insulin-secreting cells in vivo. Nat Biotechnol 2008; 26:443-452.

27 D'Amour KA, Bang AG, Eliazer S, et al. Production of pancreatic hormone-expressing endocrine cells from human embryonic stem cells. Nat Biotechnol 2006; 24:1392-1401.

28 Yang L, Soonpaa MH, Adler ED, et al. Human cardiovascular progenitor cells develop from a KDR+ embryonic-stemcell-derived population. Nature 2008; 453:524-528.

29 Zhu S, Wurdak H, Wang J, et al. A small molecule primes embryonic stem cells for differentiation. Cell Stem Cell 2009; 4:416-426.

30 Borowiak M, Maehr R, Chen S, et al. Small molecules efficiently direct endodermal differentiation of mouse and human embryonic stem cells. Cell Stem Cell 2009; 4:348-358.

31 Minami I, Yamada K, Otsuji Tomomi G, et al. A small molecule that promotes cardiac differentiation of human pluripotent stem cells under defined, cytokine- and xeno-free conditions. Cell Rep 2012; 2:1448-1460.

32 Gonzalez R, Lee JW, Schultz PG. Stepwise chemically induced cardiomyocyte specification of human embryonic stem cells. Angew Chem Int Ed Engl 2011; 50:11181-11185.

33 Stadtfeld M, Nagaya M, Utikal J, Weir G, Hochedlinger K. Induced pluripotent stem cells generated without viral integration. Science 2008; 322:945-949.

34 Okita K, Nakagawa M, Hyenjong H, Ichisaka T, Yamanaka S. Generation of mouse induced pluripotent stem cells without viral vectors. Science 2008; 322:949-953.

$35 \mathrm{Yu}$ J, Hu K, Smuga-Otto K, et al. Human induced pluripotent stem cells free of vector and transgene sequences. Science 2009; 324:797-801.

36 Kim D, Kim CH, Moon JI, et al. Generation of human induced pluripotent stem cells by direct delivery of reprogramming proteins. Cell Stem Cell 2009; 4:472-476.

37 Zhou H, Wu S, Joo JY, et al. Generation of induced pluripotent stem cells using recombinant proteins. Cell Stem Cell 2009; 4:381-384.

38 Warren L, Manos PD, Ahfeldt T, et al. Highly efficient reprogramming to pluripotency and directed differentiation of human cells with synthetic modified mRNA. Cell Stem Cell 2010; 7:618-630. 
39 Kubicek S, O'Sullivan RJ, August EM, et al. Reversal of H3K9me2 by a small-molecule inhibitor for the G9a histone methyltransferase. Mol Cell 2007; 25:473-481.

40 Shi Y, Desponts C, Do JT, Hahm HS, Scholer HR, Ding S. Induction of pluripotent stem cells from mouse embryonic fibroblasts by Oct4 and Klf4 with small-molecule compounds. Cell Stem Cell 2008; 3:568-574.

41 Shi Y, Do JT, Desponts C, Hahm HS, Scholer HR, Ding S. A combined chemical and genetic approach for the generation of induced pluripotent stem cells. Cell Stem Cell 2008; 2:525-528.

42 Feldman N, Gerson A, Fang J, et al. G9a-mediated irreversible epigenetic inactivation of Oct-3/4 during early embryogenesis. Nat Cell Biol 2006; 8:188-194.

43 Huangfu D, Osafune K, Maehr R, et al. Induction of pluripotent stem cells from primary human fibroblasts with only Oct4 and Sox2. Nat Biotechnol 2008; 26:1269-1275.

44 Huangfu D, Maehr R, Guo W, et al. Induction of pluripotent stem cells by defined factors is greatly improved by smallmolecule compounds. Nat Biotechnol 2008; 26:795-797.

45 Mali P, Chou B-K, Yen J, et al. Butyrate greatly enhances derivation of human induced pluripotent stem cells by promoting epigenetic remodeling and the expression of pluripotency-associated genes. Stem Cells 2010; 28:713-720.

46 Li W, Zhou H, Abujarour R, et al. Generation of humaninduced pluripotent stem cells in the absence of exogenous Sox2. Stem Cells 2009; 27:2992-3000

47 Onder TT, Kara N, Cherry A, et al. Chromatin-modifying enzymes as modulators of reprogramming. Nature 2012; 483:598-602.

48 Zhu S, Li W, Zhou H, et al. Reprogramming of human primary somatic cells by OCT4 and chemical compounds. Cell Stem Cell 2010; 7:651-655.

49 Zhou W, Choi M, Margineantu D, et al.HIF $1 \alpha$ induced switch from bivalent to exclusively glycolytic metabolism during ESC-to-EpiSC/hESC transition. EMBO J 2012; 31:2103-2116

50 Varum S, Rodrigues AS, Moura MB, et al. Energy metabolism in human pluripotent stem cells and their differentiated counterparts. PLoS One 2011; 6:e20914.

51 Höing S, Rudhard Y, Reinhardt P, et al. Discovery of inhibitors of microglial neurotoxicity acting through multiple mechanisms using a stem-cell-based phenotypic assay. Cell Stem Cell 2012; 11:620-632.

52 Burdon T, Stracey C, Chambers I, Nichols J, Smith A. Suppression of SHP-2 and ERK signalling promotes self-renewal of mouse embryonic stem cells. Dev Biol 1999; 210:30-43.

53 Ying QL, Wray J, Nichols J, et al. The ground state of embryonic stem cell self-renewal. Nature 2008; 453:519-523.

54 Yang W, Wei W, Shi C, et al. Pluripotin combined with leukemia inhibitory factor greatly promotes the derivation of embryonic stem cell lines from refractory strains. Stem Cells 2009; 27:383-389.

55 Hanna J, Markoulaki S, Mitalipova M, et al. Metastable pluripotent states in NOD-mouse-derived ESCs. Cell Stem Cell 2009; 4:513-524.

56 Li W, Wei W, Zhu S, et al. Generation of rat and human induced pluripotent stem cells by combining genetic reprogramming and chemical inhibitors. Cell Stem Cell 2009;
4:16-19.

57 Buehr M, Meek S, Blair K, et al. Capture of authentic embryonic stem cells from rat blastocysts. Cell 2008; 135:12871298.

58 Li P, Tong C, Mehrian-Shai R, et al. Germline competent embryonic stem cells derived from rat blastocysts. Cell 2008; 135:1299-1310.

59 Kawamata M, Ochiya T. Generation of genetically modified rats from embryonic stem cells. Proc Natl Acad Sci USA 2010; 107:14223-14228.

60 Tesar PJ, Chenoweth JG, Brook FA, et al. New cell lines from mouse epiblast share defining features with human embryonic stem cells. Nature 2007; 448:196-199.

61 Brons IG, Smithers LE, Trotter MW, et al. Derivation of pluripotent epiblast stem cells from mammalian embryos. Nature 2007; 448:191-195.

62 Hanna J, Cheng AW, Saha K, et al. Human embryonic stem cells with biological and epigenetic characteristics similar to those of mouse ESCs. Proc Natl Acad Sci USA 2010; 107:9222-9227.

63 Kim JH, Auerbach JM, Rodriguez-Gomez JA, et al. Dopamine neurons derived from embryonic stem cells function in an animal model of Parkinson's disease. Nature 2002; 418:50-56.

64 Smith JR, Vallier L, Lupo G, Alexander M, Harris WA, Pedersen RA. Inhibition of Activin/Nodal signaling promotes specification of human embryonic stem cells into neuroectoderm. Dev Biol 2008; 313:107-117.

65 Chambers SM, Fasano CA, Papapetrou EP, Tomishima M, Sadelain M, Studer L. Highly efficient neural conversion of human ES and iPS cells by dual inhibition of SMAD signaling. Nat Biotechnol 2009; 27:275-280.

66 Kim DS, Lee JS, Leem JW, et al. Robust enhancement of neural differentiation from human ES and iPS cells regardless of their innate difference in differentiation propensity. Stem Cell Rev 2010; 6:270-281.

67 Li W, Sun W, Zhang Y, et al. Rapid induction and long-term self-renewal of primitive neural precursors from human embryonic stem cells by small molecule inhibitors. Proc Natl Acad Sci USA 2011; 108:8299-8304.

68 Mao Y, Ge X, Frank CL, et al. Disrupted in schizophrenia 1 regulates neuronal progenitor proliferation via modulation of GSK3beta/beta-catenin signaling. Cell 2009; 136:1017-1031.

69 Kim WY, Wang X, Wu Y, et al. GSK-3 is a master regulator of neural progenitor homeostasis. Nat Neurosci 2009; 12:1390-1397.

70 Lin T, Ambasudhan R, Yuan X, et al. A chemical platform for improved induction of human iPSCs. Nat Meth 2009; 6:805808.

71 Esteban MA, Wang T, Qin B, et al. Vitamin C enhances the generation of mouse and human induced pluripotent stem cells. Cell Stem Cell 2010; 6:71-79.

72 Wang $\mathrm{T}$, Chen $\mathrm{K}$, Zeng X, et al. The histone demethylases $\mathrm{jhdm} 1 \mathrm{a} / 1 \mathrm{~b}$ enhance somatic cell reprogramming in a vitamin-C-dependent manner. Cell Stem Cell 2011; 9:575-587.

73 Stadtfeld M, Apostolou E, Ferrari F, et al. Ascorbic acid prevents loss of Dlk1-Dio3 imprinting and facilitates generation of all-iPS cell mice from terminally differentiated B cells. Nat Genet 2012; 44:398-405. 
74 North TE, Goessling W, Walkley CR, et al. Prostaglandin E2 regulates vertebrate haematopoietic stem cell homeostasis. Nature 2007; 447:1007-1011.

75 Goessling W, North TE, Loewer S, et al. Genetic interaction of PGE2 and Wnt signaling regulates developmental specification of stem cells and regeneration. Cell 2009; 136:11361147.

76 Ni Terri T, Rellinger Eric J, Mukherjee A, et al. Discovering small molecules that promote cardiomyocyte generation by modulating Wnt signaling. Chem Biol 2011; 18:1658-1668.

77 Andersson O, Adams Bruce A, Yoo D, et al. Adenosine signaling promotes regeneration of pancreatic $\beta$ cells in vivo. Cell Metab 2012; 15:885-894.

78 Pieper AA, Xie S, Capota E, et al. Discovery of a proneurogenic, neuroprotective chemical. Cell 2010; 142:39-51.

79 MacMillan KS, Naidoo J, Liang J, et al. Development of proneurogenic, neuroprotective small molecules. J Am Chem Soc 2011; 133:1428-1437.

80 Wang Y, Kim E, Wang X, et al. ERK inhibition rescues defects in fate specification of Nf1-deficient neural progenitors and brain abnormalities. Cell 2012; 150:816-830.

81 Christopherson KW, Hangoc G, Mantel CR, Broxmeyer HE. Modulation of hematopoietic stem cell homing and engraftment by CD26. Science 2004; 305:1000-1003.

82 Askari AT, Unzek S, Popovic ZB, et al. Effect of stromalcell-derived factor 1 on stem-cell homing and tissue regeneration in ischaemic cardiomyopathy. Lancet 2003; 362:697703.

83 Zaruba M-M, Theiss HD, Vallaster M, et al. Synergy between CD26/DPP-IV inhibition and G-CSF improves cardiac function after acute myocardial infarction. Cell Stem Cell 2009; 4:313-323. 